\title{
ADSORÇÃO DE SULFATO PELO SOLO *
}

\author{
R. A. Catani $* * *$ \\ N. A. Glória ** \\ G. C. VitTI $* * *$
}

\begin{abstract}
Avaliou-se a capacidade de retenção ou adsorção do ânsion sulfato por várias amostras de solos, mediante a agitação de dois gramas de material com $5 \mathrm{ml}$ de soluções padrões contendo quantidades crescentes de sulfato. Após um repouso durante 16 horas, procedeu-se à determinação do teor de sulfato da solução de equilíbrio. Calculou-se a quantidade de sulfato adsorvida por diferença entre a originalmente existente e a determinada na solução de equilíbrio após a agitação da suspensão do solo e repouso.

O material estudado constituiu-se de amostras de solos procedentes de vários horizontes de diversas séries do Município de Piracicaba, tratadas com carbonato de cálcio a fim de se obter uma variação relativamente ampla do $\mathrm{pH}$.

Os dados obtidos evidenciaram que a quantidade de sulfato adsorvido pelas amostras de solos estudadas aumenta com a concentração de sulfato da solução de equilíbrio e diminui à medida que se eleva o $\mathrm{pH}$.

A equação de Freundlich, na sua forma linear, traduziu de um modo adequado a dependência da quantidade de sulfato adsorvida pelo so$\operatorname{lo}(\log \underset{\mathrm{x}}{-})$ da concentração de sulfato da solução de equilíbrio ( $\log$ c) e do $\mathrm{pH}$ do solo.
\end{abstract}

\section{INTRODUÇÃO}

O enxôfre dos minerais primários apresenta-se na forma sulfetos de vários metais. Durante os processos relacionados com o intemperismo e com a formação dos solos o ânion sulfeto ( $\mathrm{S}^{2-}$ ) é transformado a sulfato ( $\left.\mathrm{SO}_{4}^{2-}\right)$.

Os vegetais absorvem o enxôfre principalmente na forma de sulfato e depois de reduzí-lo a sulfeto $\left(\mathrm{S}^{2-}\right)$ o incorporam em numerosos compostos orgânicos, dentre os quais se sobressaem os aminoácidos sulfurados, que vão integrar as proteínas.

\footnotetext{
* Entregue para publicação em 16-12-1971.

** Departamento de Química da ESALQ.

*** Bolsista da Fundação de Amparo à Pesquisa do Estado de São Paulo.

**** Agradecimentos ao Depto. de Matemática e Estatística da ESALQ. pelos cálculos no computador.
} 
Os restos vegetais sofrem decomposição no solo e o enxôfre $\left(\mathrm{S}^{2-}\right.$ ) é transformado a sulfato, passando por diversos estágios intermediários, pela ação de microrganismos (ALEXANDER, 1961; FRENEY \& STEVENSON 1966).

A concentração de suNfato em solo é muito baixa (menos de 10\% do $S$ total) quando comparada com a de enxôfre orgânico. Por outro lado, a distribuição do ânion sulfato nas fases sólida e líquida do solo depende dos fenômenos de adsorção e dessorção do citado íon. Admite-se que há um equilíbrio entre a concentração de sulfato na fase líquida e o adsorvido. Como consequiência, resulta que tanto o teor de sulfato solúvel em água, como o solúvel em várias soluções e, talvez, a quantidade disponível às plantas, sejam uma função da concentração de sulfato adsorvido.

A quantidade de sulfato, e de outros ânions, que um solo pode adsorver depende de vários fatores, tais como: natureza do solo e do seu material co'oidal, isto é, da concentração de óxidos hidratados de ferro e alumínio, $\mathrm{pH}$ do sistema e outros, conforme já foi assinalado em trabalhos anteriores (CHAO, HARWARD \& FANG, 1962 e 1964; HARWARD \& RESISENAUER, 1966; AYLMORE, KARIM \& QUIRK, 1967 ; BARROW, 1967 ; CATANI, ALCARDE \& FURLANI, 1970; CATANI, ALCARDE \& KROLL, 1971).

Neste trabalho são apresentados os dados obtidos relativos à variação da quantidade sulfato adsorvido em função da concentração de sulfato da solução de equilíbrio e do pH do solo.

\section{MATERIAL E MÉTODOS}

\section{Material}

O material utilizado constitui-se de solos do Município de Piracicaba. A descrição geral dos mesmos pode ser condensada da seguinte maneira (RANZANI, KINJO \& FREIRE, 1967):

\section{Grande Grupo}

Podzólico Vermelho Amarelo, var. Piracicaba
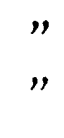

"

Latosólico Vermelho

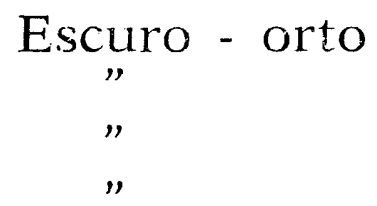

Série

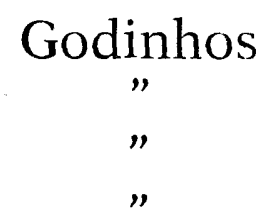

Guamium
$"$
$"$
Horizonte

Classe textural

$A p$
$A_{12}$
$A_{2}$
$A_{3} / B_{1}$

Ap

$\mathrm{B}_{1}$

$\mathrm{B}_{2}$

$\mathrm{B}_{3}$
Barro limoso
$" \quad "$
Barro - argilo-
so - arenoso

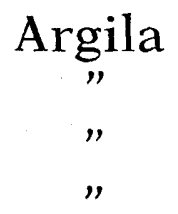


De cada horizonte dos solos descritos, foram retiradas 5 amostras de 100 gramas, que, depois de preparadas, foram tratadas com 0 (zero), 100, 200, 300 e $400 \mathrm{mg}$ de carbonato, fornecendo um total de 40 amostras (CATANI \& ALONSO, 1969).

As amostras ficaram incubadas com umidade favorável, até a estabilização do $\mathrm{pH}$ ( 40 dias ), quandio adquiriram as características descritas nos quadros $1,2,3$ e 4 .

QUADRO 1 - Principais características químicas das amostras procedentes dos horizontes Ap e $A_{12}$ da Série Godinhos, pertencente ao Podzólico Vermelho Amarelo, variação Piracicaba.

\begin{tabular}{|c|c|c|c|c|c|}
\hline \multirow{2}{*}{$\begin{array}{c}\text { Amostras } \\
\text { n. }{ }^{\circ}\end{array}$} & \multirow[b]{2}{*}{ Horizonte } & \multirow{2}{*}{$\begin{array}{c}\text { pH da Susp. } \\
\text { aquosa } \\
(1: 2,5)\end{array}$} & \multicolumn{3}{|c|}{ e. $\mathrm{mg}$ por $100 \mathrm{~g}$ de solo } \\
\hline & & & $\mathrm{Ca}^{2+*}$ & $\mathrm{Mg}^{2+*}$ & $\mathrm{~A} 1{ }^{+3}$ \\
\hline 1 & $\mathrm{Ap}$ & 4,20 & 1,40 & 0,68 & 3,80 \\
\hline 2 & & 4,75 & 3,60 & 0,67 & 1,66 \\
\hline 3 & ", & 5,25 & 5,97 & 0,88 & 0,26 \\
\hline 4 & $"$ & 6,00 & 7,62 & 0,79 & $\operatorname{tr}$ \\
\hline 5 & $"$ & 6,70 & 9,35 & 0,80 & $\mathrm{tr}$ \\
\hline 6 & $A_{1}$ & 4,60 & 1,04 & 0,56 & 3,43 \\
\hline 7 & $"$ & 5,10 & 3.29 & 0,48 & 1,27 \\
\hline 8 & $"$ & 5,75 & 5,30 & 0,43 & $\operatorname{tr}$ \\
\hline 9 & $"$ & 6,60 & 7,17 & 0,27 & tr \\
\hline 10 & $"$ & 7,10 & 8,75 & 0,37 & $\operatorname{tr}$ \\
\hline
\end{tabular}

* Em extratos com solução normal de KC1 (ZUNIGA \& CATANI 1967).

QUADRO 2 - Principais características químicas das amostras procedentes dos horizontais $A_{2}$ e $A_{3} / B_{1}$ da Série Godinhos, pertencente ao Podzólico Vermelho Amarelo, variação Piracicaba.

\begin{tabular}{|c|c|c|c|c|c|}
\hline \multirow{2}{*}{$\begin{array}{l}\text { Amostras } \\
\text { n. }\end{array}$} & \multirow[b]{2}{*}{ Horizonte } & \multirow{2}{*}{$\begin{array}{c}\text { pH da Susp. } \\
\text { aquosa } \\
(1: 2,5)\end{array}$} & \multicolumn{3}{|c|}{ e. $\mathrm{mg}$ por $100 \mathrm{~g}$ de solo } \\
\hline & & & $\mathrm{Ca}^{2+*}$ & $\mathrm{Mg}^{2+*}$ & $\mathrm{~A} 1{ }^{+3^{*}}$ \\
\hline 11 & $\mathrm{~A}_{2}$ & 4,70 & 1,05 & 0,55 & 3,34 \\
\hline 12 & $\ddot{2}$ & 5,20 & 3,12 & 0,25 & 1,40 \\
\hline 13 & $"$ & 5,95 & 4,86 & 0,21 & $\operatorname{tr}$ \\
\hline 14 & $"$ & 6,60 & 6,51 & 0,17 & $\operatorname{tr}$ \\
\hline 15 & "' & 7,30 & 8,40 & 0,21 & $\operatorname{tr}$ \\
\hline 16 & $\mathrm{~A}_{3} / \mathrm{B}_{1}$ & 4,65 & 0,84 & 0,76 & 4,80 \\
\hline 17 & , & 5,00 & 2,98 & 0,50 & 2,58 \\
\hline 18 & $"$ & 5,70 & 4,28 & 0,42 & $\mathrm{tr}$ \\
\hline 19 & $"$ & 6,10 & 6,93 & 0,38 & $\operatorname{tr}$ \\
\hline 20 & $"$ & 6,70 & 8,28 & 0,42 & $\operatorname{tr}$ \\
\hline
\end{tabular}


QUADRO 3 - Principais características químicas das amostras procedentes dos horizontes Ap e $B_{1}$ da Série Guamium pertencente ao Labosólico Vermelho Escuro-orto.

\begin{tabular}{|c|c|c|c|c|c|}
\hline \multirow{2}{*}{$\begin{array}{c}\text { Amostras } \\
\text { n. }\end{array}$} & \multirow{2}{*}{ Horizonte } & \multirow{2}{*}{$\begin{array}{c}\text { pH da Susp. } \\
\text { aquosa } \\
(1: 2,5)\end{array}$} & \multicolumn{3}{|c|}{ e. $\mathrm{mg}$ por $100 \mathrm{~g}$ de solo } \\
\hline & & & $\mathrm{Ca}^{2+*}$ & $\mathrm{Mg}^{2+*}$ & $\mathrm{~A} 1{ }^{+3^{*}}$ \\
\hline 21 & $\mathrm{Ap}$ & 4,80 & 1,23 & 0,52 & 0,76 \\
\hline 22 & & 5,50 & 3,43 & 0,41 & tr. \\
\hline 23 & $"$ & 6,10 & 4,96 & 0,46 & tr. \\
\hline 24 & $"$ & 6,55 & 6,46 & 0,47 & tr. \\
\hline 25 & $"$ & 7,20 & 7,74 & 0,40 & tr. \\
\hline 26 & $\mathrm{~B}_{1}$ & 4,75 & 2,01 & 0,58 & 0,81 \\
\hline 27 & $"$ & 5,50 & 4,17 & 0,32 & 0,10 \\
\hline 28 & $"$ & 6,00 & 6,23 & 0,33 & tr. \\
\hline 29 & $"$ & 6,50 & 7,25 & 0,60 & tr. \\
\hline 30 & $"$ & 7,10 & 8,73 & 0,48 & tr. \\
\hline
\end{tabular}

QUADRO 4 - Principais características químicas das amostras procedentes dos horizontes $\mathrm{B}_{2}$ e $\mathrm{B}_{3}$ da Série Guamium pertencente ao Latosólico Vermelho Escuro-orto.

\begin{tabular}{|c|c|c|c|c|c|}
\hline \multirow{2}{*}{$\begin{array}{c}\text { Amostras } \\
\text { n. }\end{array}$} & \multirow[b]{2}{*}{ Horizonte } & \multirow{2}{*}{$\begin{array}{c}\text { pH da Susp. } \\
\text { aquosa } \\
(1: 2,5)\end{array}$} & \multicolumn{3}{|c|}{ e. $\mathrm{mg}$ por $100 \mathrm{~g}$ de solo } \\
\hline & & & $\mathrm{Ca}^{2+*}$ & $\mathrm{Mg}^{2+*}$ & $\mathrm{~A} 1{ }^{+3^{*}}$ \\
\hline 31 & $\mathrm{~B}_{2}$ & 4,70 & 1,19 & 0,41 & 1,14 \\
\hline 32 & $"$ & 5,35 & 3,74 & 0,19 & 0,16 \\
\hline 33 & $"$ & 6,00 & 5,23 & 0,44 & $\operatorname{tr}$ \\
\hline 34 & $"$ & 6,45 & 6,84 & 0,38 & $\operatorname{tr}$ \\
\hline 35 & $"$ & 7,00 & 8,14 & 0,24 & $\mathrm{tr}$ \\
\hline 36 & $\mathrm{~B}_{3}$ & 4,85 & 0,68 & 0,32 & 1,17 \\
\hline 37 & 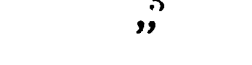 & 5,45 & 3,18 & 0,23 & 0,15 \\
\hline 38 & $"$ & 6,00 & 4,96 & 0,23 & $\operatorname{tr}$ \\
\hline 39 & $"$ & 6,50 & 6,62 & 0,14 & $\mathrm{tr}$ \\
\hline 40 & $"$ & 7,05 & 7,76 & 0,15 & $\mathrm{tr}$ \\
\hline
\end{tabular}

\section{Métodos}

Reativos

Dos reativos utilizados, merecem menção especial os seguintes:

Cloranilato de bário. Reativo foi preparado pela reação entre solução aquosa de cloreto de bário a $5 \%$ e de ácido cloranílico a $0,1 \%$ conforme técnica descrita por BERTOLACINI \& BARNEY (1957).

Solução tampão 0,1 M em ácido acético e em acetato de sódio. Preparada a partir de ácido acético glacial e acetato de sódio. 
Solução estoque de sulfato contendo $1000 \mathrm{ppm}$ de $\mathrm{SO}_{4}^{2-}$. O sulfato de potássio anidro, $\mathrm{K}_{2} \mathrm{SO}_{4}$, foi sêco a $110^{\circ} \mathrm{C}$ durante 1 hora e após esfriar, em dessecador, pesou-se $0,9070 \mathrm{~g}$ que foi transferido para balão volumétrico de $500 \mathrm{ml}$ e o volume completado com água desmineralizada.

Soluções padrões de sulfato. A partir da solução estoque, foram preparadas soluções padrões contendo $5,0-10,0-20,0-40,0-$ 80,0 e 120,0 ppm de $\mathrm{SO}_{4}^{2-}$.

Suspensão de cloranilato de bário, em solução tampão 0,01M em ácido acético e 0,01M em acetato de sódio. Um grama de cloranilato de bário, $\mathrm{BaC}_{e} \mathrm{CI}_{2} \mathrm{O}_{4}$, foi colocado em suspensão em $10 \mathrm{ml}$ de solução tampão de ácido-acético-acetato de sódio $0,1 \mathrm{M}$, e o volume foi completado a $100 \mathrm{ml}$ com água desmineralizada.

Resina Dowex 50 - X8 $(50-100$ mesh $)$, com capacidade total de troca de cátions de 5,4 e.mg por grama de resina sêca.

Preparo da curva padrão ou relação entre a concentração de $\mathrm{SO}_{4}^{2-}$ e a absorbância das soluções padrões (GLORIA \& VITTI, 1968; VITTI, 1969).

Foram passados através das colunas de resina trocadora de cátions, $2 \mathrm{ml}$ das soluções padrões de sulfato, contendo $5,0-10,0-20,0$ e 40,0 ppm de $\mathrm{SO}_{4}^{2-}$, que depois de lavadas com mais ou menos 5 $\mathrm{ml}$ de água desmineralizada, os eluídos foram recebidos em balões volumétricos de 50,0 ml. Acrescentaram-se aos balões, $25 \mathrm{ml}$ de álcool etílico, $99,5^{\circ} \mathrm{GL}, 5 \mathrm{ml}$ de suspensão de cloranilato de bário em solução de ácido acético-acetato de sódio $0,01 \mathrm{M}$ e os volumes foram completados com água desmineralizada. Após 15 minutos, intervalo nos quais as suspensões foram agitadas intermitentemente, contrífugou-se parte das suspensões (2500 rpm durante 3-4 minutos) e procedeu-se às leituras no espectofotômetro Beckman, modêlo DB a 308 milimicrons, sendo $100 \%$ de transmitância obtida com solução isenta de sulfato $\mathrm{e}$ tratada de forma similar às demais.

\section{Procedimento}

Foram pesados quatro vêzes $2,000 \mathrm{~g}$ de cada amostra de solo, transferidos para tubos de centrífuga e acrescentaram-se a cada um dêles $5 \mathrm{ml}$ de solução padrão de sulfato contendo, respectivamente, $20,0-40,0-80,0$ e $120,0 \mathrm{ppm}$ de $\mathrm{SO}_{4}^{2-}$. Os tubos foram agitados energicamente e deixados em repouso por 16 horas. Transcorrido êsse período, foi realizada nova agitação do material e procedeu-se à centrifugação a $3000 \mathrm{rpm}$, até o líquido sobrenadante permanecer límpido. Dos tubos contendo soluções com 20 e 40 ppm de $\mathrm{SO}_{4}^{2-}$ foram re- 
tiradios $2 \mathrm{ml}$ do sobrenadante; dos tubos contendo solução com 80 ppm de $\mathrm{SO}_{4}^{2-}$, foi retirado $1 \mathrm{ml}$ do sobrenadante; e dos tubos contendo solução com $120 \mathrm{ppm}$ de $\cdot \mathrm{SO}_{4}^{2-}$, foi retirado $0,5 \mathrm{ml}$ do sobrenadante. As mencionadas alíquotas foram passadas através das colunas de resina trocadora de cátions, que depois de lavadas com, aproximadamente, $5 \mathrm{ml}$ de água desmineralizada, os eluídos foram recebidos em balões volumétricos de $50 \mathrm{ml}$. Daí por diante, prosseguiu-se conforme descrito no preparo da curva padrão.

Calculou-se a quantidade de sulfato adsorvido por diferença entre a originalidade existente e a determinada na solução de equilíbrio após a agitação da suspensão do 'solo e repouso.

\section{RESULTADOS OBTIDOS E DISCUSSÃO} e 12

Os dados obtidos constam dos quadros n.os 5, 6, 7, 8, 9, 10, 11

QUADRO 5 - Adsorção de sulfato pelo horizonte Ap, da série Guamim, do Grande Grupo Latosólico Vermelho Escuro-orto, em função da concentração de sulfato da solução de equilíbrio e do pH do solo.

\begin{tabular}{|c|c|c|c|c|c|}
\hline \multirow{2}{*}{$\begin{array}{l}\text { Concentração } \\
\text { da solução } \\
\text { de equilíbrio }\end{array}$} & \multicolumn{5}{|c|}{$\mathrm{pH}$ do solo } \\
\hline & 4,80 & 5,50 & 6,10 & 6,55 & 7,20 \\
\hline $\mathrm{ug} \mathrm{SO}_{4} / \mathrm{m} 1$ & \multicolumn{5}{|c|}{ Sulfato adsorvido em $\mathrm{ug} / \mathrm{g}$ de solo ( $\mathrm{ppm}$ ) } \\
\hline 20 & 28 & 2 & - & - & - \\
\hline 10 & 52 & 21 & - & - & - \\
\hline 80 & 93 & 50 & 11 & - & - \\
\hline 120 & 125 & 70 & 47 & 24 & 24 \\
\hline
\end{tabular}

QUADRO 6 - Adsorção de sulfato pelo horizonte $B_{1}$, da série Guamim, do Grande Grupo Latosólico Vermelho Escuro-orto, em função da concentração de sulfato da solução de equilíbrio e do pH do solo.

\begin{tabular}{crrrrrc}
\hline $\begin{array}{c}\text { Concentração } \\
\text { da solução } \\
\text { de equilíbrio }\end{array}$ & 4,75 & 5,50 & 6,00 & 6,50 & 7,10 \\
\cline { 2 - 6 } & \multicolumn{5}{c}{ SH do solo } \\
\hline ug SO $_{4} / \mathrm{m} 1$ & 22 & 4 & - & - & - \\
20 & 39 & 19 & - & - & - \\
40 & 74 & 38 & 11 & - & - \\
80 & 117 & 78 & 31 & 24 & 24 \\
120 & & & Sulfato adsorvido em & ug/g de & solo & $(\mathrm{ppm})$ \\
\hline
\end{tabular}


QUADRO 7 - Adsorção de sulfato pelo horizonte $B_{2}$, da série Guamim, do Grande Grupo Latosólico Vermelho Escuro-orto, em função da concentração de sulfato da solução de equilíbrio e do $\mathrm{pH}$ do solo.

\begin{tabular}{|c|c|c|c|c|c|}
\hline \multirow{2}{*}{$\begin{array}{l}\text { Concentração } \\
\text { da solução } \\
\text { de equilíbrio }\end{array}$} & \multicolumn{5}{|c|}{$\mathrm{pH}$ do solo } \\
\hline & 4,70 & 5,35 & 6.00 & 6,45 & 7,00 \\
\hline ug $\mathrm{SO}_{4}^{-2} / \mathrm{m} 1$ & \multicolumn{5}{|c|}{ Sulfato adsorvido $\mathrm{em} \mathrm{ug} / \mathrm{g}$ de solo $(\mathrm{ppm})$} \\
\hline 20 & 44 & 21 & 20 & 8 & 0,4 \\
\hline 40 & 62 & 47 & 35 & 25 & 7 \\
\hline 80 & 120 & 89 & 81 & 54 & 34 \\
\hline 120 & 180 & 133 & 109 & 109 & 102 \\
\hline
\end{tabular}

QUADRO 8 - Adsorção de sulfato pelo horizonte $B_{3}$, da série Guamium, do Grande Grupo Latosólico Vermelho Escuro-orto, em função da concentração de sulfato da solução de equilíbrio e do pH do solo.

\begin{tabular}{|c|c|c|c|c|c|}
\hline \multirow{2}{*}{$\begin{array}{l}\text { Concentração } \\
\text { da solução } \\
\text { de equilíbrio }\end{array}$} & \multicolumn{5}{|c|}{$\mathrm{pH}$ do solo } \\
\hline & 4,85 & 5,45 & 6,00 & 6,50 & 7,05 \\
\hline ug $\mathrm{SO}_{4} / \mathrm{m} 1$ & \multicolumn{5}{|c|}{ Sulfato adsorvido em $\mathrm{ug} / \mathrm{g}$ de solo ( $\mathrm{ppm}$ ) } \\
\hline 20 & 38 & 34 & 24 & 10 & 16 \\
\hline 40 & 70 & 62 & 48 & 43 & 31 \\
\hline 80 & 120 & 113 & 93 & 85 & 74 \\
\hline 120 & 172 & 172 & 148 & 141 & 133 \\
\hline
\end{tabular}

QUADRO 9 - Adsorção de sulfato pelo horizonte Ap da série Godinhos, do Grande Grupo Podzólico Vermelho-Amarelo, variação Piracicaba em função da concentração de sulfato da solução de equilíbrio e do $\mathrm{pH}$ do solo.

\begin{tabular}{|c|c|c|c|c|c|}
\hline \multirow{2}{*}{$\begin{array}{l}\text { Concentração } \\
\text { da solução } \\
\text { de equilíbrio }\end{array}$} & \multicolumn{5}{|c|}{ pH do solo } \\
\hline & 4,20 & 4,75 & 5,25 & 6,00 & 6,70 \\
\hline ug $\mathrm{SO}_{4} / \mathrm{m} 1$ & \multicolumn{5}{|c|}{ Sulfato adsorvido em $\mathrm{ug} / \mathrm{g}$ de solo ( $\mathrm{ppm}$ ) } \\
\hline 20 & 2 & 2 & - & - & - \\
\hline 40 & 25 & 23 & - & - & - \\
\hline 80 & 42 & 38 & 23 & 19 & 11 \\
\hline 120 & 102 & 94 & 70 & 63 & 55 \\
\hline
\end{tabular}


QUADRO 10 - Adsorção de sulfato pelo horizonte $A_{12}$, da série Godinhos, do Grande Grupo Podzólico Vermelho-Amarelo, variação Piracicaba, em função da concentração de sulfato da solução de equilíbrio e do $\mathrm{pH}$ do solo.

\begin{tabular}{|c|c|c|c|c|c|}
\hline \multirow{2}{*}{$\begin{array}{l}\text { Concentração } \\
\text { da solução } \\
\text { de equilíbrio }\end{array}$} & \multicolumn{5}{|c|}{$\mathrm{pH}$ do solo } \\
\hline & 4,60 & 5,10 & 5,75 & 6,60 & 7,10 \\
\hline ug $\mathrm{SO}_{4}^{-2} / \mathrm{ml}$ & \multicolumn{5}{|c|}{ Sulfato adsorvido em ug/g de solo ( $\mathrm{ppm}$ ) } \\
\hline 20 & 16 & 8 & - & - & - \\
\hline 40 & 25 & 21 & 5 & - & - \\
\hline 80 & 58 & 54 & 38 & 3 & - \\
\hline 120 & 109 & 109 & 94 & 24 & 24 \\
\hline
\end{tabular}

QUADRO 10 - Adsorção de sulfato pelo horizonte $\mathrm{A}_{2}$, da série Godinhes, do Grande Grupo Podzólico Vermelho-Amarelo, variação Piracicaba, em função da concentração de sulfato da solução de equilíbrio e do $\mathrm{pH}$ do solo.

\begin{tabular}{|c|c|c|c|c|c|}
\hline \multirow{2}{*}{$\begin{array}{l}\text { Concentração } \\
\text { da solução } \\
\text { de equilíbrio }\end{array}$} & \multicolumn{5}{|c|}{$\mathrm{pH}$ do solo } \\
\hline & 4,70 & 5,20 & 5,95 & 6,60 & 7,30 \\
\hline ug $\mathrm{SO}_{4} / \mathrm{m} 1$ & \multicolumn{5}{|c|}{ Sulfato adsorvido em $\mathrm{ug} / \mathrm{g}$ de solo ( $\mathrm{ppm}$ ) } \\
\hline 20 & 24 & 14 & 6 & 8 & - \\
\hline 40 & 41 & 27 & 7 & 11 & - \\
\hline 80 & 63 & 54 & 50 & 34 & 18 \\
\hline 120 & 125 & 102 & 102 & 94 & 94 \\
\hline
\end{tabular}

QUADRO 12 - Adsorção de sulfato pelo horizonte $A_{3} / B_{1}$, da série Godinhos, do Grande Grupo Podzólico Vermelho-Amarelo, variação Płracicaba, em função da concentração de sulfato da solução de equilíbrio e do $\mathrm{pH}$ do solo.

\begin{tabular}{|c|c|c|c|c|c|}
\hline \multirow{2}{*}{$\begin{array}{l}\text { Concentração } \\
\text { da solução } \\
\text { de equilíbrio }\end{array}$} & \multicolumn{5}{|c|}{$\mathrm{pH}$ do solo } \\
\hline & 4,65 & 5,00 & 5,70 & 6,10 & 6,70 \\
\hline ug $\mathrm{SO}_{4}^{-2} / \mathrm{m} 1$ & \multicolumn{5}{|c|}{ Sulfato adsorvido em $\mathrm{ug} / \mathrm{g}$ de solo ( $\mathrm{ppm}$} \\
\hline 20 & 24 & 26 & 24 & 12 & 6 \\
\hline 40 & 50 & 58 & 52 & 25 & 23 \\
\hline 80 & 113 & 89 & 73 & 54 & 46 \\
\hline 120 & 172 & 172 & 156 & 109 & 94 \\
\hline
\end{tabular}


Os dados dos quadros n.os 5 a 12 evidenciam que a quantidade de sulfato ( $\mathrm{SO}_{4}^{2-}$ ) adsorvida por grama de solo ( $\mathrm{ppm}$ de sulfato adsorvido) aumenta com a elevação da concentração de sulfato da solução de equilíbrio è diminui com o aumento do $\mathrm{pH}$ do solo.

A equação de Freundlich (WEISER, 1949; GLASSTONE \& LEWIS 1960) em sua forma linear, é apresentada em 1 , onde $\frac{\mathrm{x}}{\mathrm{m}}$ repre$\mathrm{m}$ senta a quantidade em microgramas de sulfato adsorvido por grama de solo; c é a concentração de sulfato na solução de equilíbrio, e $\mathbf{n}$ e $\mathrm{K}$ são constantes.

$$
\log \frac{\mathrm{x}}{\mathrm{m}}=\mathrm{n} \log \mathrm{c}+\log \mathrm{K}(1)
$$

A equação de adsorção de Freundlich foi empregada no estudo de adsorção de sulfato porque, da mesma maneira com o que ocorreu com o estudo da adsorção de molibdato (CATANI, ALCARDE \& FURLANI, 1970) e de boro (CATANI, ALCARDE \& KROLL, 1971), a citada equação traduziu de modo mais adequado a adsorção, do que a expressão de Langmuir, nos intervalos de concentráções utilizados.

Colocando-se no eixo das abcissas o valor de $\log \mathbf{c}$ (concentração de sulfato na solução de equilíbrio) e no eixo das ordenadas os $\mathrm{x}$

respectivos valores de $\log -$, foram obtidas 27 equações de regres$\mathrm{m}$

são. Vinte e cinco apresentaram um valor para o coeficiente de regressão (r) significativo ao nível de 5\% (ou menor) de probabilidade.

Colocando-se no eixo das abcissas o valor do $\mathrm{pH}$ do solo e no eixo das ordenadas os respectivos valores de $\log -\mathbf{x}$ conservando-se $\mathrm{m}$

constante a concentração de sulfato da solução de equilíbrio, foram calculadas 19 equações de regressão. Das 19 equações, em 8 o valor de $\mathbf{r}$ foi significativo ao nível de 5\% (ou menor) de probabilidade. Em 6 equações o valor de $\mathbf{r}$ foi significativo ao nível compreendido entre 5 e $10 \%$ de probabilidade, e em 5 não foi significativo (probabilidade maior de $10 \%)$.

O comportamento do ânion sulfato, relativo à adsorção pelo solo, foi muito semelhante ao do ânion molibdato, conforme esclarecem os dados já apresentados (CATANI, ALCARDE \& FURLANI, 1970). 
Assim, de um modo geral, pode-se afirmar que o $\mathrm{pH}$ do solo exerce um efeito importante sôbre a adsorção de sulfato, isto é, aumentando-se o pH diminui a adsorção.

\section{CONCLUSÕES}

a) A adsorção de sulfato pelas amostras de solos estudadas cresce com o aumento da concentração do citado íon na solução' de equilíbrio, conservando-se constante o $\mathrm{pH}$ do solo.

b) A adsorção de sulfato pelas amostras de solos estudados decresce com o aumento do $\mathrm{pH}$ do solo, conservando-se constante a concentração de $\mathrm{SO}_{4}^{2-}$ na solução de eauilíbrio.

c) Os dados obtidos se adaptaram de um modo mais adequado à equação de adsorção de Freundlich, quando comparada com a de Langmuir, no intervalo de concentração de sulfato estudado.

\section{SUMMARY}

\section{ADSORPTION OF SULFATE BY SOILS}

The adsorption of sulfate by soil samples was studied and the data obtained showed that amount of $\mathrm{SO}_{4}^{2--}$ adsorbed increased with the equilibrium aqueous phase sulfate concentration at constant soil $\mathrm{pH}$ and decreased with soil $\mathrm{pH}$ at constante sulfate concentration of the equilibrium solution.

The linear form of the Freundlich adsorption equation expressed the relationship between $\log \frac{\mathrm{x}}{\mathrm{m}}$ and between $\log \mathbf{C}$ and soil $\mathrm{pH}$.

\section{LITERATURA CITADA}

ALEXANDER, M., 1961 - Introduction to Soil Microbiology. J. Wiley \& Sons, Inc. New York and London. Toppan Coompany, Ltd, Tokyo Japan. 472 pp.

AYLMORE, L. A. C., KARIM, M. \& QUIRK, J. P., 1967 - Adsorption and desorption of sulfate ions by soil constituentes. Soil Sci., 103:10-15.

BARROW, N. J., 1967 - Studies on the adsorption of sulfate by soils. Soil Sci., 104:342-349.

BERTOLACINI, R. J. \& J. E. BARNEY II, 1957 - Colorimetric determination of sulfate with barium chloranilate. Anal. Chem. 29:281-283. 
CATANI, R. A. \& ALONSO, O., 1969 - Avaliação de exigência de calcário do solo. Anais da E. S. A. "Luiz de Queiroz", 26:141155.

CATANI, R. A., ALCARDE, J. C. \& FURLANI, P. R., 1970 - A adsorção de molibdênio pelo solo. No prelo dos Anais da E. S. A. "Luiz de Queiroz", vol. 27(1970).

CATANI, R. A., ALCARDE, J. C. \& KROLL, F. M. 1971. Adsorção de boro pelo solo. No prelo dos Anais da E. S. A. "Luiz de Queiroz", vol. 28 (1971).

CHAO, T. T., M. E. HARWARD \& S. C. FANG, 1962 - Soil constituents and properties in the adsorption of sulfate ions. Soil Sci., 94:276-283.

CHAO, T. T., M. E. HARWARD \& S. C. FANG, 1964 - Iron or aluminum coatings in relation to sulfate adsorption characteristics of soils. Soil Sci. Soc. Am. Proc. Am. Proc., 28:632-635.

FRENEY, J. R. \& STEVENSON, F. J. 1966 - Organic sulfur transformations in soils. Soil Sci. 101:707-316.

GLóRIA, N. A., 1968 - A determinação colorimétrica do enxôfre pelo método do cloranilato de bário. Tese para obtenção do título de Docente-Livre da Cadeira n. ${ }^{\circ}$ 10, Química Analítica e Físico-Química da E. S. A. "Luiz de Queiroz", U.S.P. Piracicaba, S.P., 134 pp. (mimeografadas).

GLóRIA, N. A. da \& G. C. VITTI, 1968 - Emprêgo de coluna de resina trocadora de cátions na separação de cátions e ânions de extrato de material vegetal. Anais da E. S. A. "Luiz de Queiroz", 25:189-202.

HARWARD, M. E. \& H. M. REISENAUER, 1966 - Reaction and movement of inorganic soil sulfur. Soil Sci., 101:326-335.

RANZANI. G., FREIRE, O \& KINJO, T., 1967 - Carta de solos do Município de Piracicaba. Centro de Estudos de Solos. ESALQ, U.S.P. Piracicaba, S.P., $85 \mathrm{pp}$. (mimeografado).

VITTI, G. C., 1969 - "A determinação de sulfato pelo método colorimétrico do cloranilato de bário. Em: Relatório Geral apresentado à FAPESP 9 pp. (datilografo).

ZUÑIGA, A. A. T. \& CATANI, R. A., 1967 - Extração de diversos íons do solo com solução normal de KC1, Anais da E.S.A. "Luiz de Queiroz", 24:289-313. 
